\title{
SPECTRAL ANALYSIS OF FRICATIVES IN VOWEL CONTEXT
}

\author{
C.H. SHADLE, C.U. DOBELKE* and C. SCULLY** \\ Department of Electronics and Computer Science, University of Southampton, GB-Southampton \\ SO9 5 NH, Great-Britain \\ *Preussen Electra, Hanover, Germany \\ ${ }^{* *}$ Department of Psychology, University of Leeds, Great-Britain
}

Ensemble averages were performed on spectra of repeated $/ \mathrm{pV}_{1} \mathrm{FV}_{2} /$ tokens in order to test the quasi-stationary assumption on which the more typical time-averaging was based. Although centered ensemble-averaged spectra were similar to time averages, ensemble averaging was then used to focus on the details of spectral change throughout a fricative, and to establish the effects of the vowel context. The main feature observed during the fricative proper was an increase in high-frequency energy in its center. In the transition regions more differences were noted in both formant transitions and the spectral shape of the noise; these differences were more noticeable for the weak fricatives and appeared to distinguish $/ \mathrm{f} / \mathrm{and}$ $/ \theta /$.

Des moyennes d'ensemble ont été effectuées sur les spectres de logatomes $/ \mathrm{pV}_{1} F V_{2} /$ répétés, afin de tester l'hypothèse de quasi-stationarité à la base des traditionnelles moyennes temporelles. Bien que les moyennes d'ensemble donnent des résultats semblables aux moyennes temporelles, lorsque'elles s'appliquent au centre, elles furent ensuite employées pour étudier en détail l'évolution du spectre tout au long de la fricative, et mettre en évidence l'effet du contexte vocalique. La principale caractéristique observée sur la fricative elle-même fut un accroîssement de l'énergie des hautes fréquences dans la région centrale. Sur les régions transitoires, d'autres différences furent trouvées tant sur les transitions formantiques que sur le spectre du bruit; ces différences étaient plus marqués pour les fricatives faibles et semblent pouvoir distinguer le $/ \mathrm{f} / \mathrm{du} / \theta /$.

\section{Introduction}

Since fricatives are inherently noisy, their broad spectral characteristics can only be observed if some form of averaging is used. The technique most often employed [1,2], called time averaging, involves placing windows consecutively (or with some overlap) throughout the duration of the fricative, computing the spectrum for each window, and averaging all the spectra together at each frequency to produce a single spectrum with reduced variance. This technique is based on the assumption that the signal is stationary within the analyzed section. For sustained fricatives this assumption is probably valid; for VCV contexts, it is probably not, since rapid articulatory and aerodynamic changes are well documented in the vowel-to-fricative and fricative-to-vowel transition regions $[3,4]$.

An alternative exists, known as ensemble averaging. Here an ensemble of signals is used; each signal in the ensemble is assumed to be produced under the same conditions even though these conditions 

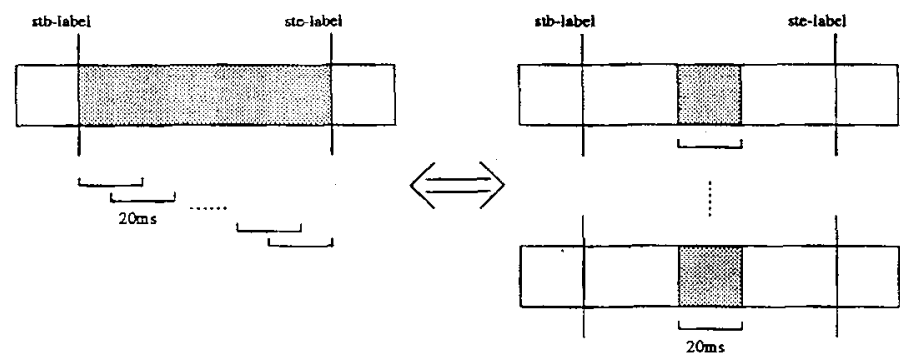

Figure 1: Diagram showing the placement of labels and windows in each fricative. The stb-label defines the beginning of the stationary portion of the fricative; ste-label, the end. Time averaging of one token is shown on the left; ensemble averaging of eight tokens, on the right.

may vary over time. Averaging is not done over time, but at the same time across the ensemble. The variance of the averaged power spectrum decreases as the number of averages increases, for either type of averaging [5]. However, the duration of the part of a single signal incorporated into an average differs greatly in the two types of averaging.

The assumptions underlying an ensemble occur rarely in spontaneous speech. However, they could be met through the use of a corpus already in existence, which is described in the next section. This corpus allowed us to compare time- and ensemble-averaged spectra, thus checking the assumptions on which each are based; to inspect spectral changes throughout the vowel-to-fricative and fricative-to-vowel transitions; and to look for evidence of difference between fricatives, not only in steady-state spectral shape, but in the patterns of the transitions and the degree of influence of the adjacent vowels.

\section{Recording Method}

The corpus used was developed jointly at the University of Leeds, University of Southampton, and the Institute Communication Parlée, I.N.C.P., Grenoble. In this corpus the nonsense word $/ \mathrm{pV}_{1} \mathrm{FV}_{2} /$ was repeated 10 to 13 times during a single breath. This set of repetitions was called an item. For each item, $V_{1}$ and $V_{2}$ were chosen from $/ a, i, u / ; F$ was one of $/ f, v, \theta, \delta, s, z, \int, Z, \xi, j, x, \gamma, h /$. Within each item, the first two and last two tokens of $/ \mathrm{pV} V_{1} \mathrm{FV}_{2} /$ were discarded, leaving 6 to 9 relatively uniform tokens forming the ensemble. Two speakers were recorded speaking this corpus while a variety of acoustic, articulatory and aerodynamic measurements were made. For this study, recordings by the first author, a native woman speaker of General American, were used. The main results shown here were based on spectral analysis of the high-fidelity acoustic recordings, which were made in an anechoic chamber using a Bruel \& Kjaer $41651 / 2$ " microphone located $1 \mathrm{~m}$ in front of the subject's mouth. Recordings were made with a Sony PCM system at 16 bits with a sampling frequency of $44.1 \mathrm{kHz}$. A calibration signal was recorded to allow absolute pressure level to be retained. These results were also compared with separately obtained recordings using a Rothenberg mask and oral pressure measurement, and electropalatography (details are given in [6]).

\section{Analysis Method}

During speech editing, each token was labelled at the beginning and end of each transition region into and out of the fricative. The cues used to locate the labels included changes in the overall amplitude of the waveform, the beginning or ending of voicing, the beginning or ending of noise, and changes in the amplitude of the first or second formant. Consistency of these cues was checked by comparing durations of the transition regions across tokens, and also by comparing with articulatory data gathered in separate recording sessions. The labels were then used to locate a $20 \mathrm{~ms}$ Hanning window at the same point in each of the tokens of a single item, in order to compute the ensemble average; for the time average, the labels within a single token defined the beginning and end of the region over which to place the analysis windows. The labelling method and placement of windows is shown schematically in Fig. 1. 

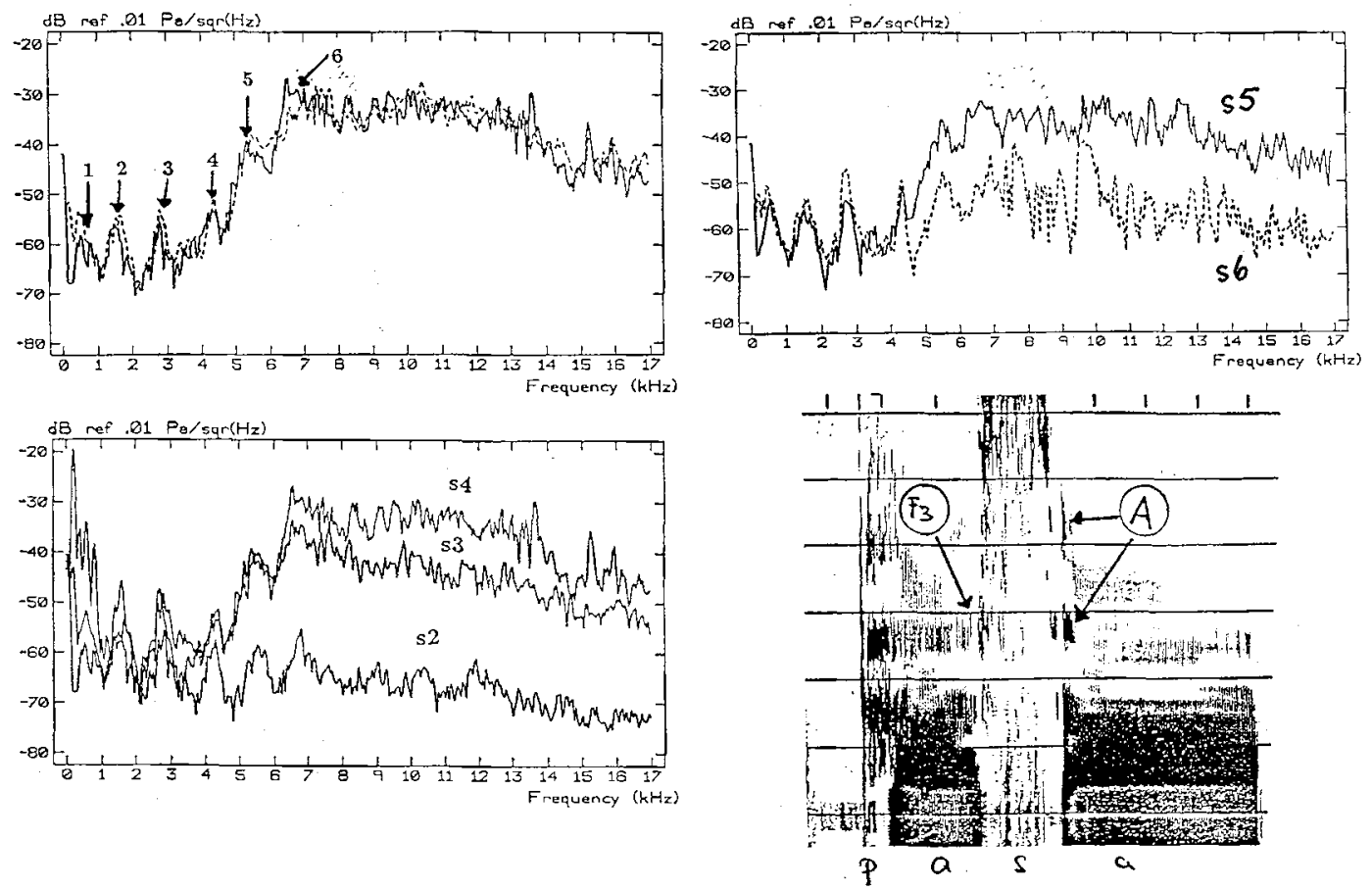

Figure 2: Spectral analysis of /pasa/. a) Time-averaged spectrum (dotted line) and ensemble average at fricative centers (solid line). b),c) Ensemble averages: $s 2$ is at end of vowel-fricative transition, s3 at start of fricative steady-state, $s 4$ in fricative center, $s 5$ and $s 6$ are adjacent, just before fricative-vowel transition. d) Spectrogram of token 3. 'A' marks aspiration noise at fricative end.

\section{Results}

Figure $2 \mathrm{a}$ contrasts time- and ensemble-averaging for $/ \mathrm{s} / \mathrm{in} / \mathrm{pasa} /$. The great similarity evidenced here holds for nearly all the fricatives examined; exceptions have an obvious explanation, such as one or two tokens that are devoiced versions of a voiced fricative, or have an audible change in amplitude. It might seem, then, that the stationary region is indeed stationary and thus that the assumption underlying time-averaging is valid. Figures $2 b$ and $2 c$ show that this is not the case: there is a clear difference between ensemble averages taken at the beginning, middle and end of the "stationary" region. The center of the fricative has the greatest energy at high frequencies and the lowest energy in the 0 to $4 \mathrm{kHz}$ range. Harmonics due to voicing die out between $\mathrm{s} 2$ and $\mathrm{s} 3$, and formants shift noticeably. What seems likely is that these changes occur during a relatively small portion of the "stationary" region, and so they are obscured by the time average.

Figure 2d shows a spectrogram of one token of /pasa/, allowing us to observe the formant transitions more closely. The second formant begins to increase well in advance of the fricative, but it and the third formant continue to shift slightly after voicing has ceased. The decrease in energy at low frequencies is clearly visible on the spectrogram; comparison with articulatory and airflow data indicates that there are three major adjustments that must be made in the transition region. The airflow increaases; the vocal folds are abducted, and they stop vibrating; the tongue must move to form the constriction. The latter takes the longest, so as a result frication begins while the constriction area is still decreasing. As the area grows smaller the air velocity through the constriction increases, which boosts the high-frequency energy and simultaneously cancels low-frequency back cavity formants more effectively $[6,7,8]$. Different vowel contexts affect the formant trajectories into and out of the $/ \mathrm{s} /$ (or $/ \int /$, but do not substantially alter the sequence or timing of events.

For the weak fricatives, however, the differences are more marked. Figure 3 contrasts /pafa/ and 

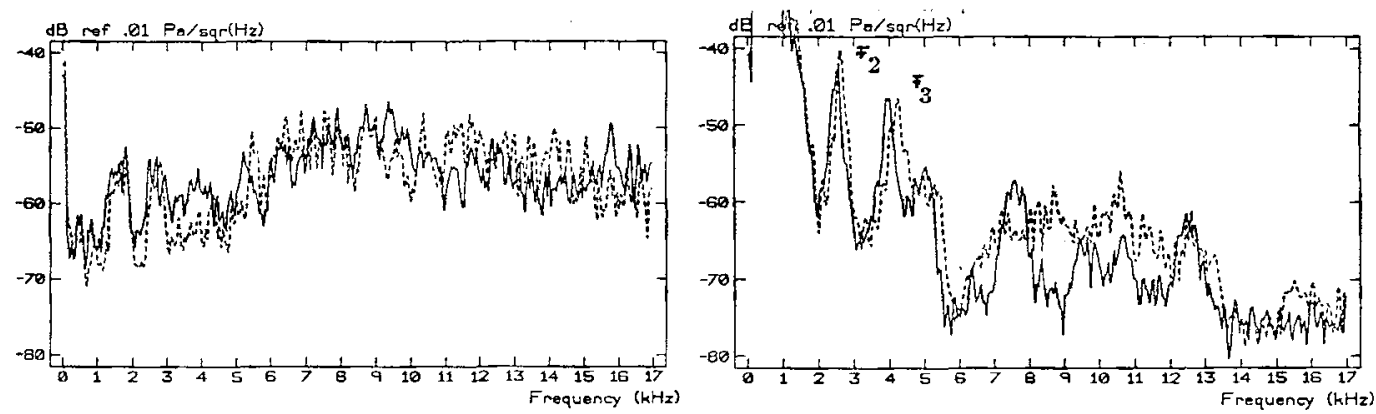

Figure 3: Ensemble averages of /pafa/ (solid lines) and / pa $\theta \mathrm{a} /$ (dotted lines). Left: center of fricatives. Right: beginning of fricative-vowel transition.

$/ \mathrm{pa} \theta \mathrm{a} /$ at two points during the fricatives. In the center of the steady-state region, the two fricatives are very similar, as has been noted in numerous studies $[7,8]$. In the transition to the vowel, however, /pa $\theta \mathrm{a} /$ retains energy in the range 7 to $12 \mathrm{kHz}$ for longer than /pafa/. Formants F2 and F3 are also significantly higher, supporting the generally accepted view [9] that the difference between these fricatives lies in the formant transitions. A similar difference between the two is observed in the transition into the fricative.

Contrasting /pifi/ and /pi $\theta \mathrm{i} /$ likewise reveals similarity in the center of the fricative, and differences in the transition regions. In this case, however (not shown), formants are lower for $/ \mathrm{pi} \theta \mathrm{i} /$. Also, the boost in energy at high frequencies does not occur in /pi $\theta \mathrm{i} /$ until much later than in $/ \mathrm{pa} \theta \mathrm{a} /$. A possible explanation is that since the tongue tip is required to form the constriction for $/ \theta /$ but not for $/ f /$, formants will shift during $/ \theta /$ transitions. The direction of shift will depend on the vowel. The timing differences in boosting of high-frequency energy are then likely due to sequencing differences that result when the tongue has a long versus a short distance to travel.

\section{Conclusion}

Ensemble averages were performed on repeated $/ \mathrm{pV}_{1} \mathrm{FV}_{2} /$ tokens in order to focus on the details of spectral change throughout a fricative, and to establish the effects of the vowel context. Ensemble averages computed in the center of the ensemble of fricatives were in general quite similar to time averages computed throughout single fricatives. However, ensemble averages at beginning and end of a fricative did differ significantly from the central averages; in particular, amplitude at high frequencies was highest in the center of the fricatives. The transition regions showed the influence of the adjacent vowel, as expected, but this influence was more marked in the case of the weak fricatives, $/ f, v, \theta, \delta /$.

\section{Acknowledgement}

This work was supported in part by a collaborative EC SCIENCE award, CEC-SCI*0147C(EDB).

\section{References}

/1/ HUGHES, W. and HALLE, M. J. Acoust. Soc. Am. 28 (1956) 303-311.

/2/ HEINZ, J.M. and STEVENS, K.N. J. Acoust. Soc. Am. 33 (1961) 589-596.

/3/ KLATT, D. H. J. Acoust. Soc. Am. 67 (1980) 971-995.

/4/ SCULLY, C. In Speech Production and Speech Modelling, W.J. Hardcastle and A. Marchal (eds.), Kluwer (1990) 151-186. Kluwer

/5/ BENDAT, J.S. and PIERSOL, A.G. Random Data, Wiley-Interscience (1971).

/6/ SCully, C., GeORGeS, E. and CASTelli, E. Proc. Xith Int. Cong. Phon. Aix-en-Provence (1991) 58-61.

17/ SHADLE, C. H. Tech. Rpt. 506, Res. Lab. Elec., M.I.T. (1985).

/8/ SHADLE, C.H. In Speech Production and Speech Modelling, W.J. Hardcastle and A. Marchal (eds.), Kluwer (1990), 187-209.

19/ HARRIS, K.Lang. and Speech 1 (1959) 1-7. 